\title{
Analytic theory of optical nanoplasmonic metamaterials
}

\author{
Angela Demetriadou* and Ortwin Hess ${ }^{\dagger}$ \\ Blackett Laboratory, Department of Physics, Imperial College London, London SW7 2AZ, United Kingdom
}

(Received 22 November 2012; revised manuscript received 13 February 2013; published 4 April 2013)

\begin{abstract}
Recent advances in nanofabrication techniques allow for the manufacture of optical metamaterials, bringing their unique and extraordinary properties to the visible regime and beyond. However, an analytical description of optical nanoplasmonic metamaterials is challenging due to the characteristic optical behavior of metals. Here we present an analytical theory that allows us to bring established microwave metamaterials models to optical wavelengths. This method is implemented for nanoscaled plasmonic wire-mesh and trihelical metamaterials, and we obtain an accurate prediction for their dispersive behavior at optical and near-IR wavelengths.
\end{abstract}

DOI: 10.1103/PhysRevB.87.161101

The metamaterial paradigm "function from structure" has in recent years opened the door to exciting new materials with unprecedented properties, such as negative refractive index. ${ }^{1}$ Moreover, in combination with transformation optics, it allowed a conception of dramatic functionalities, such as perfect lensing, ${ }^{1}$ cloaking, ${ }^{2,3}$ and broadband slow and stopped light. ${ }^{4}$ As the meta-atoms that make up a metamaterial characteristically consist of metal structures that are smaller than the wavelength of light, metamaterial principles such as invisibility cloaking ${ }^{2,3}$ were first experimentally demonstrated in the microwave regime. Recent advances in nanofabrication methods, particularly bottom-up and self-assembly techniques, now allow for the design and manufacture of bulky artificial metallic structures with nanoscaled and complex structural features ${ }^{5-7}$ forming nanoplasmonic metamaterials operating at optical (near-infrared and visible) frequencies. These new fabrication techniques have so far allowed the manufacture of bulk metamaterials with a periodicity of $50 \mathrm{~nm}$ (Refs. 6 and 7) and most recently as small as $35 \mathrm{~nm}$ (Ref. 8), which are well below the optical wavelength.

In the conception of metamaterials, analytical theories have been the trailblazers for their design and opened our understanding of their physical properties. For chiral metamaterials this has been particularly successful in the microwave region. $^{9-11}$ At optical wavelengths, the forces exerted by incident light fields on the nearly free (conduction) electrons of a metal lead to collective electron-photon oscillations that typically occur on scales of just a few tens of nanometers or less. These collective electron-photon oscillations can either be localized [localized surface plasmons (LSPs)] or propagating [surface plasmon polaritons (SPPs)], forming areas of high intensity around and inside the plasmonic nanoparticles, the meta-atoms. In addition to nanolocalization of incident light waves, surface plasmons may efficiently allow for the reverse effect, i.e. the outcoupling to the far-field continuum of the near field of emissive molecules and quantum dots placed adjacently to the metal metamolecule, thereby acting as nanoantennas. Clearly, analytical metamaterials models that are very appropriate for microwaves fall short of describing the optical response of nanoscaled metallic structures and nanoplasmonic metamaterials. Indeed, it was found through experimental results and numerical calculations ${ }^{12,13}$ that the actual performance of these structures is significantly red shifted compared to what one may have expected from their geometrical parameters. ${ }^{14}$
PACS number(s): 81.05.Xj, 33.55.+b, 78.20.Ek, 78.67.Pt

In this Rapid Communication, we develop an analytic theory for the optical properties of nanoplasmonic metamaterials built up from generalized metal nanowires and propose a robust self-consistent method that can be used to bring well-established analytical metamaterial models from the microwave domain to optical wavelengths. In particular, we will demonstrate the significance of our theory in bringing the celebrated "wire-mesh" metamaterial model ${ }^{15,16}$ to the optical frequency domain with a nanoscale wire mesh and present an analytical model for trihelical nanoplasmonic metamaterials. Note that the applicability of our analytical method has no restriction within the realms of the metamaterial field, particularly since the wavelength is always larger than the metamaterial's unit cell.

Let us start by focusing on a thin straight and infinitely extended metal nanowire of radius $r_{w}$ and permittivity $\varepsilon_{m}$ that is embedded in a dielectric host medium with permittivity $\varepsilon_{h}$, as shown in Fig. 1. The skin depth $\delta$ is dependent on the properties of the metal and is given by $\delta=\sqrt{\left(2 / k_{0}^{2} \mu_{m}\right)\left[\varepsilon_{m}^{\prime \prime}-i\left(1-\varepsilon_{m}^{\prime}\right)\right] /\left[\left(\varepsilon_{m}^{\prime \prime}\right)^{2}+\left(1-\varepsilon_{m}^{\prime}\right)^{2}\right]}$, where $k_{0}$ is the wave number in free space and $\mu_{m}$ the magnetic permeability of the metal. At microwave frequencies, any current induced on a metallic wire will flow on its surface, producing a magnetic effect around the wire and there would be no field inside the metal. Now, at optical regimes, the fields penetrate in the metal (since $r_{w}<\delta$ ), and they induce an electron flow throughout the metallic nanowire and not just on its surface. The current flowing at the center of the nanowire induces a magnetic field in a loop enclosing the current, as shown in Fig. 1. Of course, the magnitude of the induced magnetic field increases with the amount of metal it encloses, and therefore it reaches a maximum value just outside the metallic nanowire. Now these fields inside the metal slow down the effective/overall electron flow, which is modeled in this Rapid Communication with the wave number $\gamma$, and significantly red shift the effective response of the nanowire.

Hence,the wave vector describing the electron flow is $k_{0} \sqrt{\varepsilon_{m}}$ and the effect of plasmon polarities (i.e., charge distribution inside the metal) is taken on board the wave vector $\gamma$, such that the overall field propagation inside the wire is governed by ${ }^{14} \kappa_{1}=\sqrt{k_{0}^{2} \varepsilon_{m}-\gamma^{2}}$. The fields in the dielectric host medium are affected in a similar way by the plasmonic excitation, and therefore we have $\kappa_{2}=\sqrt{k_{0}^{2} \varepsilon_{h}-\gamma^{2}}$. Hence, $\gamma$ dominates the red shift to the dispersive behavior of nanometallic structures from the $\omega$ frequency predicted from 


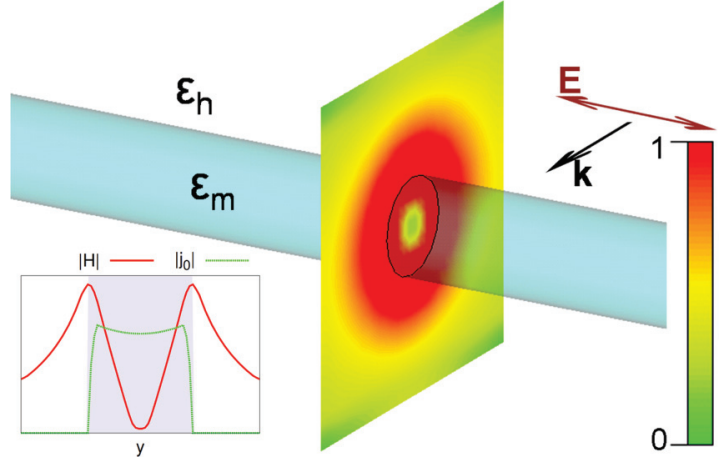

FIG. 1. (Color online) A nanowire made from metal of permittivity $\varepsilon_{m}$ and surrounded by a host medium of $\varepsilon_{h}$ is completely penetrated by an incident electric field $E$. The currents induced inside the wire give rise to a magnetic field $|H|$ shown at the cross-section surface of the wire. The amplitude of $|H|$ and the current density $\left|j_{0}\right|$ inside the wire are also displayed along a line parallel to the $y$ axis and passing though the center of the wire (blue-shaded area represents the metallic wire).

microwave analytical models to $\omega^{\prime}$ that is actually observed from experimental results and numerical calculations.

The relationship between the two frequencies $\left(\omega\right.$ and $\left.\omega^{\prime}\right)$ is therefore dependent on $\gamma$ as ${ }^{14}$

$$
\omega=\frac{\omega^{\prime}}{\left(\frac{k_{0}}{\gamma}\right)-\frac{4 r_{w} \omega^{\prime}}{2 \pi c_{0}}},
$$

where the only unknown parameter is $\gamma$. Here, we find an exact solution for $k_{0} / \gamma$, and then we replace all $\omega$ parameters in established microwave analytical models for wire-mesh and trihelical metamaterials with (1), to obtain their optical dispersive behavior.

In order to find $\gamma$, we observe the field behavior in Fig. 1, which is identical to the $T M_{0}$ mode field profile for a cylindrical dielectric waveguide. The nanowire thus effectively acts as a cylindrical dielectric waveguide since it allows the field to completely penetrate it. Hence, we use the waveguide theory to model the field penetration and plasmonic excitation for a metal nanowire, and we find $\gamma$ by solving ${ }^{17}$

$$
\frac{\varepsilon_{m}}{\kappa_{1} r_{w}} \frac{J_{1}\left(\kappa_{1} r_{w}\right)}{J_{0}\left(\kappa_{1} r_{w}\right)}=\frac{\varepsilon_{h}}{\kappa_{2} r_{w}} \frac{H_{1}^{(1)}\left(\kappa_{2} r_{w}\right)}{H_{0}^{1}\left(\kappa_{2} r_{w}\right)},
$$

where $J_{n}$ and $H_{n}^{(1)}$ are the cylindrical Bessel and Hankel functions of the first kind. For thin wires (i.e., $\lambda \gg r_{w}$ ) the Bessel and Hankel functions are expanded to first order in $\kappa r_{w}$ terms, which is a condition always true for metamaterials where $\lambda>a$ ( $a$ is the periodic unit cell of the metamaterial). Therefore, the above equation can be reduced to ${ }^{14}$

$$
z^{2}+a(z)+\frac{2 \varepsilon_{h}}{\varepsilon_{m}}\left(\frac{2 a(z)-2 a(z)^{2}-1}{4 a(z)}\right)=0,
$$

where $a(z)=\Gamma-\ln (2)+\ln |z|$, and $\Gamma=0.577$ is the Euler constant. We approximate $\ln |z| \sim 2\left(\frac{z-1}{z+1}\right)$, which is valid for $0.5<|z|<3$. Therefore, $a(z) \simeq a_{0}+2\left(\frac{z-1}{z+1}\right)$, where

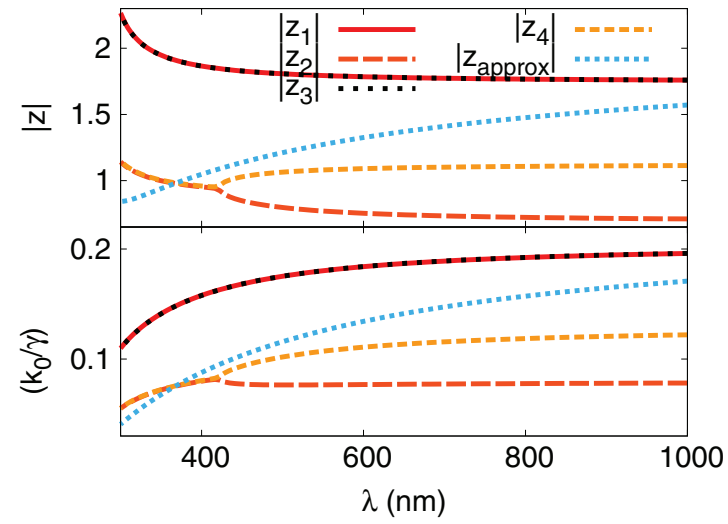

FIG. 2. (Color online) The four solutions $\left|z_{i}\right|$ and their corresponding $\left(k_{0} / \gamma\right)$ are plotted for Drude-silver metal, with the approximated solution $\left|z_{\text {approx }}\right|$ of Novotny, which ignores metallic losses.

$a_{0}=\Gamma-\ln (2)$. Also, where $z$ (Ref. 14):

$$
z=\sqrt{\frac{2 \varepsilon_{h}}{-\varepsilon_{m}}}\left(\frac{k_{0} / \gamma}{k_{0} r_{w} \sqrt{1-\varepsilon_{h}\left(k_{0} / \gamma\right)^{2}}}\right)=\sqrt{\frac{2 \varepsilon_{h}}{-\varepsilon_{m}}} \widetilde{z} .
$$

There are four solutions to (3), plotted in Fig. 2 for silver, which have a rather long and complex format:

$$
\begin{aligned}
& z_{1,2}=-\frac{a_{0}}{2\left(2+a_{0}\right)}-\frac{1}{2} \sqrt{A} \mp \frac{1}{2} \sqrt{B-C}, \\
& z_{3,4}=-\frac{a_{0}}{2\left(2+a_{0}\right)}+\frac{1}{2} \sqrt{A} \mp \frac{1}{2} \sqrt{B+C},
\end{aligned}
$$

where

$$
\begin{gathered}
A=\left(\frac{a_{0}}{2+a_{0}}\right)^{2}-\frac{J}{2\left(2+a_{0}\right) \varepsilon_{m}}+H, \\
B=2\left(\frac{a_{0}}{2+a_{0}}\right)^{2}-\frac{J}{2\left(2+a_{0}\right) \varepsilon_{m}}-H, \\
C=-8\left(\frac{a_{0}}{2+a_{0}}\right)\left(A-H+\frac{G}{a_{0} \varepsilon_{m}}\right),
\end{gathered}
$$

and $\quad J=3 \varepsilon_{m}\left(2+5 a_{0}+a_{0}^{2}\right)-\varepsilon_{h}\left(5+6 a_{0}+2 a_{0}^{2}\right), \quad G=$ $2 \varepsilon_{m}\left(a_{0}+2\right)\left(a_{0}-2\right)-\varepsilon_{h}\left(2 a_{0}^{2}-2 a_{0}-7\right), \quad$ and $H$ is a complex function of $\varepsilon_{h}$ and $\varepsilon_{m}$, whose full format is shown in the Supplemental Material. ${ }^{18}$ In Fig. 3, function $H$ is plotted for different metals and host media. We observe that for frequencies where the real part of the metal's permittivity is $\left(\varepsilon_{m} / \varepsilon_{h}\right)<-5, H$ is independent of the metal's and host medium's material properties. Therefore, to slightly simplify solutions in (5), $H$ can safely be approximated by $H=-1.537+i 0.658712$ for wavelengths where $\left(\varepsilon_{m} / \varepsilon_{h}\right)<-5$. The four solutions of $|z|$ are plotted in Fig. 2 for a Drude-like silver of $\varepsilon_{\infty}=4.028$, plasma frequency $\omega_{m p}=1.39 \times 10^{16} \mathrm{rad} / \mathrm{s}$, and collision frequency $g=3.14 \times 10^{13} \mathrm{~s}^{-1}$. From Fig. 2 , one can see that $\left|z_{1}\right|$ and $\left|z_{3}\right|$ are always degenerate. On the other hand, $\left|z_{2}\right|$ and $\left|z_{4}\right|$ are only degenerate for wavelengths where $\left(\varepsilon_{m} / \varepsilon_{h}\right)>-5$. Finally, we note that all our solutions are within the approximation limit $0.5<|z|<3$ that we took earlier for $\ln |z|$.

Using Eqs. (1) and (4), we can now obtain $k_{0} / \gamma$ that is also plotted in Fig. 2 for all four $z$ solutions. Finally, the frequency shift occurring in dispersive metallic media, when they are 


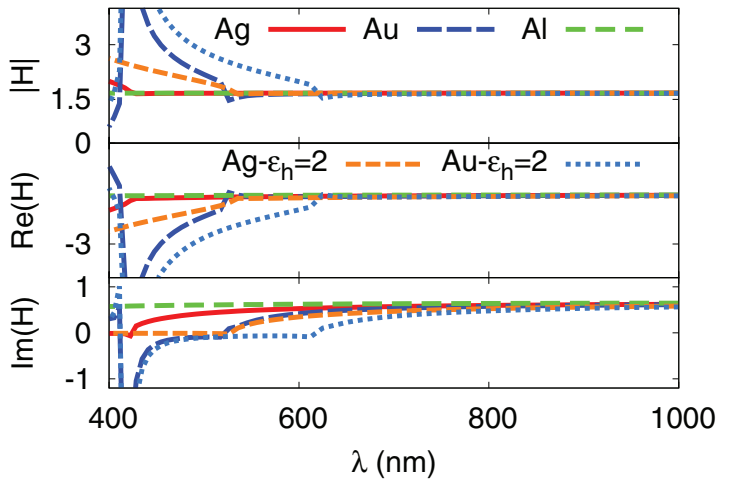

FIG. 3. (Color online) The $H$ function against the wavelength for silver and gold $\left(\omega_{m p}=1.3544 \times 10^{16} \mathrm{rad} / \mathrm{s}, g=1.5367 \times 10^{14}\right.$ $\left.\mathrm{s}^{-1}, \varepsilon_{\infty}=9.0685\right)$ in a vacuum and $\varepsilon_{h}=2$, and aluminium $\left(\omega_{m p}=\right.$ $\left.2.295 \times 10^{16} \mathrm{rad} / \mathrm{s}, g=1.27 \times 10^{14} \mathrm{~s}^{-1}, \varepsilon_{\infty}=0.8476\right)$.

completely penetrated by electromagnetic waves is given by

$$
\omega=\frac{\omega^{\prime}}{\sqrt{\frac{\left(\omega^{\prime} r_{w} \widetilde{z}\right)^{2}}{c_{0}^{2}+\varepsilon_{h}\left(\omega^{\prime} r_{w} \widetilde{z}\right)^{2}}-\frac{4 r_{w} \omega^{\prime}}{2 \pi c_{0}}},}
$$

where $\omega$ is the frequency found in any microwave analytical dispersive model and $\omega^{\prime}$ is the new frequency accounting for the field penetration and plasmonic excitation of metals at optical frequencies. Note that $\tilde{z}$ is dispersive [i.e., $\widetilde{z}\left(\omega^{\prime}\right)$ ] since it is a function of $\varepsilon_{m}$. Equation (9) can now be implemented easily into any established microwave analytical metamaterial model, bringing it to optical wavelengths. Finally, (9) is valid for metal nanostructures of circular cross section. If one wishes to obtain the relationship between $\omega$ and $\omega^{\prime}$ for metal nanostructures of square or orthogonal cross sections, then (2) needs to be rewritten accordingly (and follow the same methodology). In this Rapid Communication, we choose to derive (9) for circular cross sections, since at the nanometer scale metal structures most commonly have approximately a circularlike cross section.

Novotny in Ref. 14 derived an approximate solution for (5) assuming lossless Drude metals. His approximate solution $\widetilde{z}_{\text {approx }}=\alpha_{1}+\alpha_{2} \frac{\omega_{p m}}{\omega}$ is also plotted in Fig. 2 (blue dotted line) with our exact solutions. The approximation provides an indication for the order of magnitude of the frequency shift for simple metallic designs, such as a dipole optical antenna as it was intended to do (where $\omega_{p m}$ is the plasma frequency of the metal and $\alpha_{1}$ and $\alpha_{2}$ are coefficients given in Ref. 14).

We note that despite the fact that Novotny's approximate solution ${ }^{14}$ gives quite reasonable results for simple systems, such as optical dipole antennas (rods of short length), it cannot predict the properties of complex metallic geometries, in particular, metamaterials that quite often are complex designs of several wavelengths in size (i.e., wire-mesh, trihelical, and gyroidal metamaterials). Furthermore, losses are an important aspect for metamaterial performance and cannot be neglected. ${ }^{19}$ In contrary to $\widetilde{z}_{\text {approx }}$, our exact solution in (5) accounts for metallic losses that can significantly affect the performance of optical metamaterials. However, the most important advantage of our model is that it allows for any metal (not just Drude-metals) to be considered and in fact experimental data (in the form of a fitting function) to be used for $\varepsilon_{m}$ to even account for intersubband transitions. Additionally, the model described in this Rapid Communication can be also applied for both inhomogeneous and nonlinear host media (i.e., gain and Kerr media), by simply expressing $\varepsilon_{h}$ with the appropriate function. The derived analytical formulation in this Rapid Communication unveils the physics governing the electromagnetic interaction of metal nanostructures with light in the infrared and visible regimes and consequently, modeling the homogenized electromagnetic behavior of optical metamaterials.

To demonstrate the generality of our theory on analytic methods, we now apply it to two different nanoplasmonic metamaterials, the wire mesh ${ }^{15,16,20}$ and the trihelical metamaterial (THM). ${ }^{11}$

The classic wire-mesh metamaterial is composed of thin wires aligned with the three orthogonal axes [as shown in the inset of Fig. 4(b)] and behaves as an artificial plasma. ${ }^{15,16,20}$ Its effective electric permittivity obeys the Drude model:

$$
\varepsilon_{w m}=1-\frac{\omega_{p-w m}^{2}}{\omega^{2}+i \gamma \omega},
$$

where $\omega_{p-m p}$ is the cutoff frequency given by ${ }^{15,16,20} \omega_{p-w m}=$ $c_{0} \sqrt{2 \pi /\left\{a^{2}\left[\ln \left(\sqrt{\frac{a^{2}}{\pi r_{w}^{2}}}\right)+0.5275\right]\right\}}$. Note that we omitted $\varepsilon_{h}$ in the above formula, since it is taken into account from our model in (9). Now, if $r_{w} \leqslant \delta$, we substitute $\omega$ in (10) with (9). Assuming silver nanowires of radius $r_{w}=3.5 \mathrm{~nm}$ arranged in a periodic square lattice of $a=40 \mathrm{~nm}$, we calculate $\varepsilon_{w m}$ using (9) and (10) and they are plotted in Fig. 4(a) with numerical calculations ${ }^{21}$ for comparison. The numerical values were retrieved from S-parameter calculations, using the method described in Ref. 22. We see a good agreement between the

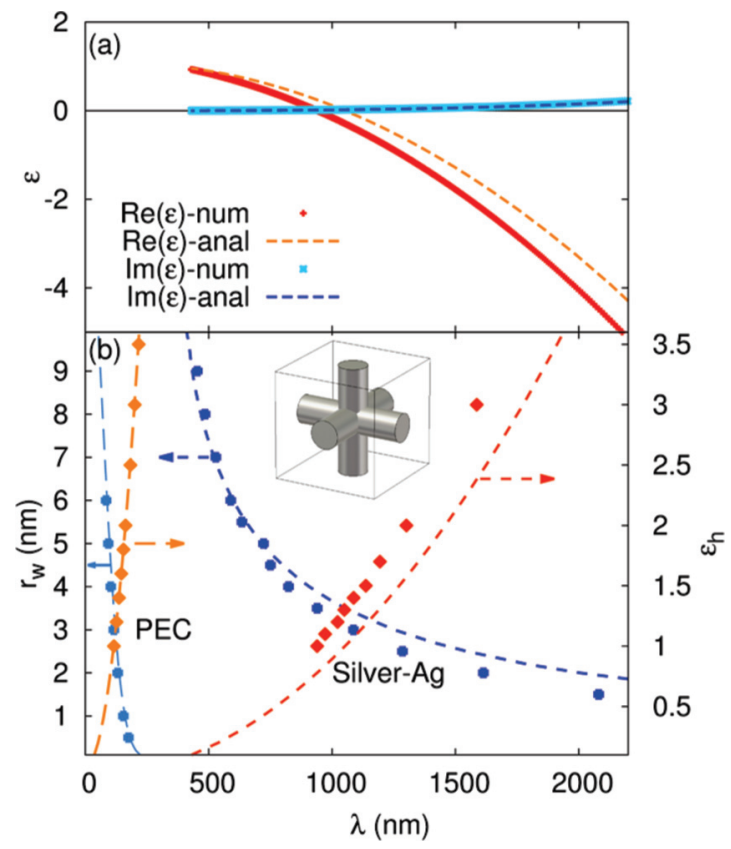

FIG. 4. (Color online) (a) The analytical prediction (lines) and numerical calculations (points) for a silver wire mesh with $r_{w}=$ $3.5 \mathrm{~nm}$ and periodicity $a=40 \mathrm{~nm}$. (b) The cutoff frequency variation for different $r_{w}$ and $\varepsilon_{h}$ of perfect electric conductor (PEC) and silver wire-mesh nanometamaterial. 


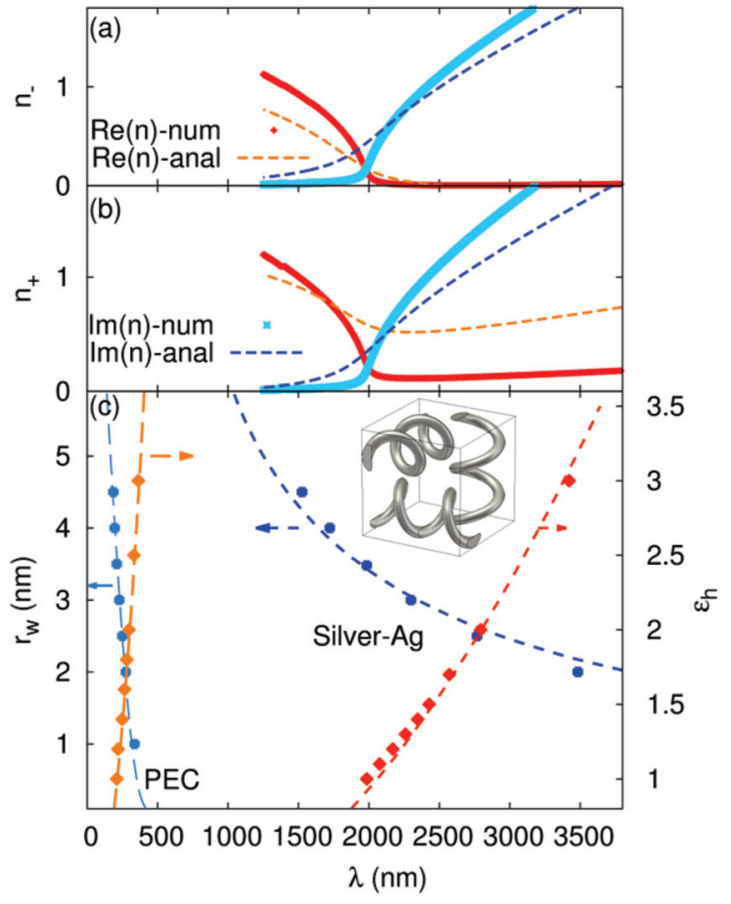

FIG. 5. (Color online) (a) The $n_{-}$and (b) $n_{+}$of a THM of $r_{w}=3.48 \mathrm{~nm}$, pitch $p=20 \mathrm{~nm}$, helix radius $R=6.52 \mathrm{~nm}$, and periodicity $a=40 \mathrm{~nm}$. (c) The cutoff frequency variation for different $r_{w}$ and $\varepsilon_{h}$ of perfect electric conductor (PEC) and silver wire-mesh nanometamaterial.

two over a large wavelength spectrum. It can be shown that the small discrepancies for higher $\varepsilon_{h}$ are due to the initial analytical model in (10), and scale with the wavelength. Note that for $\tilde{z}$ in (9), we used solution $z_{4}$, since $z_{1}, z_{2}$, and $z_{3}$ gave unphysical electromagnetic properties for the optical wire-mesh metamaterial. In Fig. 4(b), we plot the analytical prediction (lines) of the wavelength where $\varepsilon_{w m}=0$ for various nanowire radii $r_{w}$ in different host media $\varepsilon_{h}$ that show remarkable agreement with numerical calculations (points). It is noteworthy that the wiremesh cutoff frequency of extremely thin wires significantly shifts from a few tens of nanometers for PEC metal to a few micrometers for silver. Also, small variations on the optical wire radius produce extreme shifts for the cutoff frequency and our model predicts very accurately this dramatic behavior.

To further demonstrate the applicability of our method on any nanoplasmonic metamaterial, we now apply it to a chiral metamaterial. The THM was introduced in Ref. 11, as an analytic model for a complex nanoscale self-assembled metamaterial, such as the metallic gyroids. ${ }^{12}$ The THM is composed of three wire helices oriented along the three orthogonal axes [as shown in the inset of Fig. 5(c)]. Hence, as for the wire-mesh metamaterial, the THM shows a Drude behavior for the effective electric permittivity:

$$
\chi_{E E}^{-1}=\left(\frac{a}{l}\right)\left(\frac{\omega^{2}+i \Gamma \omega}{\omega^{2}+i \Gamma \omega-\omega_{p}^{2}}\right),
$$

where $\omega_{p}=c_{0} \sqrt{p /\left\{\pi R^{2} a^{2}\left[1-\pi R^{2} / a^{2}-L p /(2 \pi R)\right]\right\}}, \Gamma$ is a loss parameter, and $L$ is the self-inductance of the helical wires (see Supplemental Material ${ }^{23}$ and Ref. 11 for more details). Unlike the wire mesh, the effective magnetic response $\chi_{H H}$ of the THM is resonant and not always equal to 1 . However, the most important characteristic of the THM is that it exhibits chiral components $\left(\kappa_{E H}\right.$ and $\left.\kappa_{H E}\right)$, which in this case $\kappa_{E H}=-\kappa_{H E}$. Due to chirality, left-handed and right-handed circularly polarized waves propagating in a THM will experience different refractive indices, given by $n_{ \pm}=1 /\left(\sqrt{\chi_{E E}^{-1}(\omega) \chi_{H H}^{-1}(\omega)} \pm i \kappa_{E H}^{-1}(\omega) / c_{0}\right)$.

The electromagnetic response of a nanoscaled THM, where metallic components are completely penetrated by the incident electromagnetic fields, is derived by replacing all $\omega$ terms in (11), $\chi_{H H}$ and $\kappa_{E H}$ (see Supplemental Material ${ }^{24}$ ) with (9). Again, only the $z_{4}$ solution gives physical results. In Figs. 5(a) and 5(b), we plot the analytical predictions for $n_{-}$and $n_{+}$, respectively, of a silver THM (with dimensions $r_{w}=3.48 \mathrm{~nm}, p=20 \mathrm{~nm}, R=6.52 \mathrm{~nm}$, and $a=40 \mathrm{~nm}$ ), along with numerical calculations that show remarkable agreement. The numerical calculation values were retrieved from S-parameter calculations, but using a different method as described in Ref. 25, due to the presence of chirality. As before, we change the geometrical parameters of the THM by varying the wire's radius $r_{w}$ and the host medium $\varepsilon_{h}$. The wavelength where $\chi_{E E}=0$ for different structures is plotted in Fig. 5(c), where again the analytical (lines) and numerical (points) calculations show notable agreement. Remarkably, as for the wire-mesh metamaterial, we observe a dramatic wavelength shift between the optical and microwave models for extremely small geometrical changes in the structure.

In summary, we have derived an analytical theory for nanoplasmonic metamaterials that accounts for the field penetration and plasmonic excitation of nanoscaled metallic geometries. Recent advances in fabrication techniques of self-assembled metal nanostructures have led to the successful manufacture of bulk three-dimensional (3D) nanoplasmonic metamaterials with periodicity of just a few nanometers, operating at optical wavelengths. Our proposed model allows for the accurate analytical description of these optical nanoplasmonic metamaterials, whose behavior is dictated by the complex optical behavior of metals. Here, we have established that our analytical method transfers well-accepted microwave analytical models for metamaterials to optical wavelengths. In fact, our theory accounts for metallic losses, which dominate the behavior of optical metamaterials, any metallic dispersive behavior, and even intersubband transitions, through the use of experimental data models for the metal permittivity. This method is a valuable tool to analytically predict the optical behavior of any metamaterial and coupled optical nanoantennas, but most importantly complex selfassembled nanoplasmonic metamaterials, such as gyroidal structures.

\footnotetext{
*a.demetriadou06@imperial.ac.uk

†o.hess@imperial.ac.uk

${ }^{1}$ J. B. Pendry, Phys. Rev. Lett. 85, 3966 (2000).
}

${ }^{2}$ J. B. Pendry, D. Schurig, and D. R. Smith, Science 312, 1780 (2006).

${ }^{3}$ D. Schurig, J. J. Mock, B. J. Justice, S. A. Cummer, J. B. Pendry, A. F. Starr, and D. R. Smith, Science 314, 977 (2006). 
${ }^{4}$ K. L. Tsakmakidis, A. D. Boardman, and O. Hess, Nature (London) 450, 397 (2007).

${ }^{5}$ J. K. Gansel, M. Thiel, M. S. Rill, M. Decker, K. Bade, V. Saile, G. von Freymann, S. Linden, and M. Wegener, Science 325, 1513 (2009).

${ }^{6}$ I. Vukovic, S. Punzhin, Z. Vukovic, P. Onck, J. M. DeHosson, G. Brinke, and K. Loos, ACS Nano 5, 6339 (2011).

${ }^{7}$ S. Vignolini, N. A. Yufa, P. S. Cunha, S. Guldin, I. Rushkin, M. Stefik, K. Hur, U. Wiesner, J. J. Baumberg, and U. Steiner, Adv. Mater. 24, OP23 (2012).

${ }^{8}$ Stefano Salvatore, Angela Demetriadou, Silvia Vignolini, Sang Soon Oh, Sebastian Wuestner, Nataliya A. Yufa, Morgan Stefik, Ulrich Wiesner, Jeremy J. Baumberg, Ortwin Hess, and Ullrich Steiner, Advanced Materials (to be published).

${ }^{9}$ A. Demetriadou and J. B. Pendry, J. Phys.: Condens. Matter 21, 376003 (2009).

${ }^{10}$ A. Demetriadou and J. B. Pendry, Physica B: Condens. Matter 405, 2943 (2010).

${ }^{11}$ A. Demetriadou, S. S. Oh, S. Wuestner, and O. Hess, New J. Phys. 14, 083032 (2012).

${ }^{12}$ K. Hur, Y. Francescato, V. Giannini, S. A. Maier, R. G. Henning, and U. Wiesner, Angewandte Chemie 50, 11985 (2011).

${ }^{13}$ S. S. Oh, A. Demetriadou, S. Wuestner, and O. Hess, Adv. Mater. 25, 612 (2013).

${ }^{14}$ L. Novotny, Phys. Rev. Lett. 98, 266802 (2007).
${ }^{15}$ J. B. Pendry, A. J. Holden, D. J. Robbins, and W. J. Stewart, J. Phys.: Condens. Matter 10, 4785 (1998).

${ }^{16}$ A. Demetriadou and J. B. Pendry, J. Phys.: Condens. Matter 20, 295222 (2008).

${ }^{17}$ Robert E. Collin, Field Theory of Guided Waves (Wiley-Blackwell, New York, 1991).

${ }^{18}$ See Supplemental Material at http://link.aps.org/supplemental/ 10.1103/PhysRevB.87.161101 for full format of equation (5).

${ }^{19}$ O. Hess, J. B. Pendry, S. Maier, R. F. Oulton, J. M. Hamm, and K. L. Tsakmakidis, Nat. Mater. 11, 573 (2012).

${ }^{20}$ P. A. Belov, R. Marques, S. I. Maslovski, I. S. Nefedov, M. Silveirinha, C. R. Simovski, and S. A. Tretyakov, Phys. Rev. B 67, 113103 (2003).

${ }^{21}$ Simulation calculations were performed using finite-integration techniques (CST MICROWAVE STUDIO).

${ }^{22}$ D. R. Smith, D. C. Vier, T. Koschny, and C. M. Soukoulis, Phys. Rev. E 71, 036617 (2005).

${ }^{23}$ See Supplemental Material at http://link.aps.org/supplemental/ 10.1103/PhysRevB.87.161101 for definition of the parameters used for the THM model.

${ }^{24}$ See Supplemental Material at http://link.aps.org/supplemental/ 10.1103/PhysRevB.87.161101 for description and definition of the THM model.

${ }^{25}$ R. Zhao, T. Koschny, and C. M. Soukoulis, Opt. Express 18, 14553 (2010). 